\title{
Monocyte activation in patients with Wegener's granulomatosis
}

\author{
Anneke C Muller Kobold, Cees G M Kallenberg, Jan Willem Cohen Tervaert
}

\begin{abstract}
Objective-Wegener's granulomatosis (WG) is an inflammatory disorder characterised by granulomatous inflammation, vasculitis, and necrotising vasculitis and is strongly associated with anti-neutrophil cytoplasmic antibodies (ANCA). Activated monocytes/macrophages are present in renal biopsy specimens and participate in granuloma formation by synthesising and secreting a variety of chemoattractants, growth factors, and cytokines. In view of these findings, in vivo monocyte activation was evaluated in patients with WG and the findings related to parameters of clinical disease activity.
\end{abstract}

Methods-Monocyte activation was analysed by measuring plasma concentrations of soluble products of monocyte activation, that is neopterin and interleukin 6 (IL6), by ELISA, and by quantitating the surface expression of activation markers on circulating monocytes by flow cytometry.

Results-Twenty four patients with active WG were included in this study. Ten of these patients were also analysed at the time of remission. Twelve patients with sepsis served as positive controls, and $\mathbf{1 0}$ healthy volunteers as negative controls for monocyte activation. Patients with active disease had increased monocyte activation compared with healthy controls as shown by increased concentrations of neopterin $(p<0.0001)$ and increased surface expression of CD11b $(p<0.05)$ and CD64 ( $p<0.05)$. In those patients with increased concentrations of IL6 during active disease plasma concentrations of IL6 decreased during follow up when patients went into remission $(p<0.0001)$. In addition, neopterin $(r=0.37, r=0.44)$, IL6 $(r=0.37, r=0.60)$ and CD63 expression $(r=0.39, r=0.45)$ correlated significantly with disease activity as measured by the Birmingham Vasculitis Activity Score and $C$ reactive protein values, respectively. Compared with patients with sepsis, all markers of monocyte activation

Department of Clinical Immunology,

University Hospital Groningen, PO Box 30001, 9700 RB Groningen, the Netherlands

Correspondence to: Dr A C Muller Kobold.

Accepted for publication 11 January 1999
Wegener's granulomatosis (WG) is an inflammatory disorder of presumed autoimmune ori- gin characterised by granulomatous inflammation of the respiratory tract, necrotising vasculitis, and glomerulonephritis. This disease is strongly associated with anti-neutrophil cytoplasmic antibodies (ANCA). ANCA in this disorder are directed against proteinase 3 (Pr3), myeloperoxidase (MPO), and/or, occasionally, human leucocyte elastase (HLE). ${ }^{1}$

Although the pathophysiological role of ANCA in WG has not yet been fully elucidated, several lines of evidence suggest that ANCA are involved in the development of WG. Firstly, relapses of WG are frequently preceded by a rise in ANCA titre and can be prevented by treatment based on changes in those titres. ${ }^{2}$ Secondly, in vitro, ANCA have the potential to activate primed neutrophils to the production of reactive oxygen species and the release of lytic enzymes, ${ }^{34}$ and to activate and damage cultured endothelial cells. ${ }^{5-7}$ Priming with tumour necrosis factor $\alpha(\mathrm{TNF} \alpha)$ and cytochalasin $\mathrm{B},{ }^{34}$ or $\mathrm{TNF} \alpha$ in combination with interleukin $8,{ }^{8}$ induces surface expression of ANCA antigens on the neutrophil cellsurface, which become then available for interaction with ANCA. Subsequently, ANCA can interact with their respective antigens as well as with $\mathrm{Fc}$ receptors, especially the second $\mathrm{Fc}$ receptor, on the neutrophil cellsurface, which leads to neutrophil activation. ${ }^{9} 10$

Most studies have focused on the capacity of ANCA to induce neutrophil activation. Apart from neutrophils, however, monocytes/ macrophages also play a pivotal part in lesions development in WG. Monocytes/macrophages are found in renal biopsy specimens from patients with WG. ${ }^{11}$ Activated monocytes/ macrophages participate in particular in granuloma formation, characteristically found in patients with WG, by synthesising and secreting a variety of chemoattractants, growth factors and cytokines. ${ }^{12}$ Furthermore, monocytes/macrophages may induce vascular damage by the production and secretion of reactive oxygen radicals and lytic proteases. Monocytes also contain the ANCA antigens Pr3 and MPO in their granules. Like neutrophils, they express ANCA antigens upon priming, ${ }^{13}$ which subsequently may initiate a process of cell activation similar as has been described for neutrophils after interaction with ANCA. Indeed, it has been demonstrated that monocytes produce increased concentrations of monocyte chemoattractant protein $1(\mathrm{MCP} 1)^{14}$ or interleukin $8,{ }^{13}$ or both, upon stimulation with ANCA.

As WG is a systemic disease, several groups have hypothesised that neutrophils from patients with WG are already activated in the circulation. ${ }^{8}{ }^{15}$ Recently, Haller et al suggested 
that monocytes are also activated in the circulation. ${ }^{16}$ They demonstrated increased integrin expression on both circulating neutrophils and monocytes from patients with active WG. They did not, however, investigate the expression of ANCA antigens on circulating monocytes nor did they investigate monocyte activation in relation to disease activity.

In view of the recent in vitro data showing that ANCA can activate monocytes, we evaluated in vivo monocyte activation in patients with WG by analysing the levels of soluble products of monocyte activation, such as interleukin 6 (IL6) and neopterin, in the plasma of patients with WG. These soluble products of monocyte activation reflect the state of activation of both tissue monocytes/macrophages as well as circulating monocytes. In addition, we measured the expression of monocyte activation markers and ANCA antigens on circulating monocytes. We related the results to parameters of clinical disease activity to define their clinical relevance.

\section{Methods}

PATIENTS AND CONTROLS

The patient group consisted of 24 consecutive patients admitted to our hospital or seen at the outpatient clinic with a diagnosis of WG. Twelve consecutive patients with sepsis served as positive controls for leucocyte activation. Sepsis was defined as previously described. ${ }^{17}$ Healthy laboratory personnel served as normal controls.

DIAGNOSTIC CRITERIA

The diagnosis of WG was established according to clinical and histological criteria ${ }^{18}$ and all patients fulfilled the American College of Rheumatology (ACR) criteria for this disease..$^{19}$ Patients with active disease were grouped as: (a) patients with newly developed disease $(\mathrm{N})$, and (b) patients with relapsing disease (R). Relapsing disease was defined as previously described. $^{20}$ Table 1 gives the criteria for relapsing disease. Patients with newly developed disease were studied before treatment was started. Patients with relapsing disease were studied before treatment was instituted or intensified. Complete remission was defined as the absence of signs or symptoms attributable to active vasculitic disease.

DISEASE ACTIVITY SCORING

Disease activity was measured according to the Birmingham Vasculitis Activity Scoring index

Table 1 Criteria for relapsing disease ${ }^{20}$

Manifestations have to be new or recurrent findings:

(a) Progressive glomerulonephritis, that is, a decrease in renal function of $30 \%$ or more within 3 months in combination with (microscopic) haematuria or evidence of acute necrotising lesions on renal biopsy.

(b) Pulmonary infiltrates with or without cavitation with rising $\mathrm{C}$ reactive protein concentrations either with necrotising granulomatous inflammation or necrotising vasculitis on biopsy, or-when no histological proof can be obtained-after exclusion of infection and malignancy.

(c) Sinusitis, otitis, ulceration of nasal mucosa, or a nasal proliferative mass, in combination with necrotising granulomatous inflammation on biopsy.

(d) Miscellaneous: progressive mononeuritis multiplex, cranial nerve palsy, cerebral vasculitis, necrotising scleritis, orbital pseudotumor, progressive tracheal stenosis with active disease on biopsy, peripheral gangrene, necrotising vasculitis of medium sized or small muscular arteries.
(BVAS), ${ }^{21}$ whereas damage was measured according to the Vascular Damage Index. ${ }^{22}$

ANCA DETECTION

ANCA were detected by indirect immunofluorescence on ethanol fixed granulocytes as previously described ${ }^{23}$ using FITC labelled goat antihuman IgG (Dako, Denmark) in a 1:50 dilution. Test or control serum samples were tested in a dilution of 1:20, and further at twofold dilutions. Slides were read by two independent observers, and a titre $\geqslant 40$ was considered positive.

The specificity of ANCA for either Pr3, MPO or HLE was detected by capture ELISA as previously described. ${ }^{24}$

Anti-lactoferrin antibodies were detected by ELISA on plates directly coated with lactoferrin (5 $\mu \mathrm{g} / \mathrm{ml}$, Sigma, St Louis, MA, USA) as described. ${ }^{25}$ Serum samples were considered positive for one of the aforementioned specificities when values exceeded the mean +2 standard deviations of normal controls $(n=50)$.

\section{ANALYSIS OF MONOCYTE ACTIVATION}

Neopterin ELISA

Values of neopterin, a plasma marker for monocyte activation, ${ }^{26-29}$ were measured by using a commercially available ELISA (Brahms Diagnostica, Germany). In brief, serum samples were mixed with the neopterin/alkaline phosphatase conjugate in uncoated microtitre plates. This mixture was then transferred to a sheep polyclonal anti-neopterin coated microtitre plate and incubated for two hours at room temperature in the dark. The wells were then washed thoroughly and the substrate (4nitrophenylphosphate) incubated for $30 \mathrm{~min}$ utes at room temperature. Finally, the reaction was stopped by the addition of $2 \mathrm{M} \mathrm{NaOH}$. Plates were read at $405 \mathrm{~nm}$. As reference, a standard curve, ranging from $2-250 \mathrm{nmol} / 1$ was used. Linear range of the neopterin ELISA ranged from 2 to $250 \mathrm{nmol} / 1$. The sensitivity of this test is $2 \mathrm{nmol} / 1$.

\section{Interleukin 6 ELISA}

IL6 concentrations, a parameter for monocyte activation, ${ }^{28}{ }^{29}$ were analysed by ELISA, according to Helle et $a{ }^{\beta 0}$ with modifications. In brief, microtitre plates (Costar nr 9018, the Netherlands) were coated overnight at room temperature in phosphate buffered saline (PBS) with a monoclonal antibody against human IL6 (CLB.MIL6/16, Central Laboratory of Blood transfusion services (CLB), Amsterdam, the Netherlands) at a dilution of 1:500. After washing with $0.025 \mathrm{M}$ TRISHCL, $0.15 \mathrm{M} \mathrm{NaCl}$ and $0.05 \%$ Tween 20, plates were blocked with $2 \%$ bovine serum albumin (BSA) $/ 0.05 \%$ Tween 20 in PBS for one hour at room temperature. As reference, recombinant IL6 was used (highest concentration: $2000 \mathrm{pg} / \mathrm{ml}$ ). Subsequently, patient and healthy control plasma samples (twofold dilutions) were incubated for two hours. Wells were then washed and a biotinylated sheep polyclonal antibody against human IL6 was added (CLB.SIL6-D, CLB, Amsterdam) at a dilution of 1:3000, one hour at room temperature in the 
dark. IL6 was then detected by the addition of streptavidin poly-horseradish peroxidases (CLB, M2032 STREPTA-E+) for 30 minutes, room temperature, in the dark. Finally, plates were washed and the colour reaction was initiated by the addition of substrate (tetra methyl benzidine (TMB), and $\mathrm{H}_{2} \mathrm{O}_{2}, 20$ minutes). The colour reaction was stopped by adding 100 $\mu \mathrm{l} /$ well of $1 \mathrm{M} \mathrm{H}_{2} \mathrm{SO}_{4}$. Plates were then scanned at $450-575 \mathrm{~nm}$. Linear range of the IL6 ELISA ranged from 15 to $2000 \mathrm{pg} / \mathrm{ml}$. The sensitivity of this test is $15 \mathrm{pg} / \mathrm{ml}$.

\section{Monocyte surface marker analysis by flowcytometry}

To avoid in vitro activation of leucocytes we used a whole blood method ${ }^{31}$ as described previously. ${ }^{32}$ In brief, EDTA anticoagulated blood was kept on ice until sample preparation. Sample preparation was started always within five minutes after blood sampling. All steps were performed in Hanks's balanced salt solution (HBSS) without calcium and magnesium (Gibco, Life Technologies Ltd, Paisley, Scotland, UK), supplemented with $1 \% \mathrm{BSA}$, Boseral, Organon Teknika, Boxtel, the Netherlands). Cells were fixed with $1 \%$ paraformaldehyde in PBS for 10 minutes on ice, washed, followed by two times erythrocyte lysis with lysis buffer $\left(155 \mathrm{mM} \mathrm{NH}_{4} \mathrm{Cl}, 10 \mathrm{mM} \mathrm{KHCO}_{3}\right.$, $0.1 \mathrm{mM} \mathrm{Na} \mathrm{NDDT}_{2} \mathrm{H}_{2} \mathrm{O}$ ) for five minutes at $37^{\circ} \mathrm{C}$. A panel of monoclonal antibodies to leucocyte surface antigens was used for the analysis of monocyte activation (table 2). ${ }^{33}$ The first antibody was incubated for one hour at $4^{\circ} \mathrm{C}$. After washing, the cells were incubated with a goat antimouse Ig polyclonal antibody conjugated with phycoerythrin (Southern Biotechnology Associates Inc, Birmingham, USA), supplemented with 5\% normal goat serum and 5\% normal human serum, 1:20 diluted, for 30 minutes at $4^{\circ} \mathrm{C}$ in the dark. Subsequently, cells were washed and stored until flow cytometric analysis was performed.

Analysis of surface marker expression was performed on a Coulter Epics ELITE flow cytometer (Coulter, Hiaelea, Florida, USA), the same day or occasionally the next day (within 18 hours). When the cell pellet contained erythrocytes, the intercalating dye,
LDS751 (Exiton Chemical, Dayton, Ohio, USA) was added before flow cytometric measurement. Erythrocytes could successfully be excluded from the leucocyte population in the LDS751/forward scatter dotplot, when combined with a lifegate. Monocytes were identified by forward and sideward scatter. Data were analysed using Immuno-4 software. ${ }^{34}$

In the first experiments, QC3 beads (Flow Cytometry Standards, Leiden, the Netherlands) were used to calibrate the flow cytometer. However, these beads appeared not to be stable during the study period. In addition, batch to batch quality varied remarkably. Therefore, we decided to compare the results obtained in patients with the results obtained in healthy, age matched volunteers that were measured simultaneously.

The expression of surface markers was calculated as a mean fluorescence intensity (MFI), corrected for non-specific binding of an irrelevant antibody and the conjugate (NSB), in combination with the percentage of positive cells (pos \%). Data were expressed as a percentage of the value obtained from the healthy control who was tested in parallel, according to the following formula:

$$
\begin{aligned}
& \text { expression index } \\
& =\frac{(\mathrm{MFI}-\mathrm{NSB})_{\text {patient }} \mathrm{X} \text { pos } \%_{\text {patient }}}{(\mathrm{MFI}-\mathrm{NSB})_{\text {control }} \mathrm{X} \text { pos } \%_{\text {control }}} \times 100 \%
\end{aligned}
$$

To assess the variability of the normal population, 10 controls were analysed simultaneously. Their individual data were expressed as a percentage of the mean of the healthy control population.

OTHER ASSAYS

$C$ reactive protein $(\mathrm{CRP})$ concentrations were measured by using a particle enhanced nephelometric method and NA latex CRP reagents (Behring, Marburg, Germany).

\section{STATISTICAL ANALYSIS}

Groups were analysed for differences in surface expression by means of the Kruskal-Wallis test. Subsequently, differences between groups were analysed by the Mann-Whitney test. Correla-

\begin{tabular}{|c|c|c|c|c|}
\hline Marker & Cellular distribution & Remarks & Monoclonal antibody & Source \\
\hline $\mathrm{MPO}$ & $\begin{array}{l}\text { neutrophils } \\
\text { monocytes }\end{array}$ & increased expression after priming & 4.15 & CLB \\
\hline $\operatorname{Pr} 3$ & $\begin{array}{l}\text { neutrophils } \\
\text { monocytes }\end{array}$ & increased expression after priming & 12.8 & CLB \\
\hline HLE & $\begin{array}{l}\text { neutrophils } \\
\text { monocytes }\end{array}$ & increased expression after priming & NP57 & Dako \\
\hline CD63 & $\begin{array}{l}\text { monocytes } \\
\text { granulocytes } \\
\text { activated platelets }\end{array}$ & GP53, increased expression after priming & CLB-gran/12,435 & CLB \\
\hline CD11b & $\begin{array}{l}\text { monocytes } \\
\text { macrophages } \\
\text { granulocytes } \\
\text { NK cells }\end{array}$ & $\begin{array}{l}\text { increased expression after priming, subunit } \\
\text { of beta2 integrin CD18/ CD11b (Mac-1), } \\
\text { ligand for ICAM-1, } \beta \text { glucan, fibrinogen, } \\
\text { C3bi. }\end{array}$ & 2LPM19c & Dako \\
\hline CD49d & $\begin{array}{l}\text { monocytes } \\
\text { lymphocytes } \\
\text { eosinophils }\end{array}$ & $\begin{array}{l}\text { adhesion molecule, subunit of beta } 1 \text { integrin } \\
\text { CD29/CD49d (VLA-4). }\end{array}$ & $\mathrm{HP} 2 / 1$ & Imm. tech \\
\hline Fc $\gamma$ RI (CD64) & $\begin{array}{l}\text { monocytes } \\
\text { granulocytes }\end{array}$ & $\begin{array}{l}\text { increased expression after activation, } \\
\text { constitutively expressed on monocytes, }\end{array}$ & 22 & Medarex \\
\hline
\end{tabular}

Table 2 Activation markers on monocytes

CLB; Central Laboratory for the Bloodtransfusion Service, Amsterdam, the Netherlands, Dako; Dakopatts, Glostrup, Denmark, Imm. tech, Immunotech, Marseilles, France; Medarex, Annandale, USA 
Table 3 Clinical and serological findings in 24 patients with $W G$

\begin{tabular}{|c|c|c|c|c|c|c|c|c|c|c|c|}
\hline Patient & Age & Sex & Diagnosis & State & Titre & Spec. & $B V A S$ & $C R P$ & $V D I$ & $W B C$ & $\begin{array}{l}\text { Immunosuppressive } \\
\text { treatment at time of analysis }\end{array}$ \\
\hline \multirow[t]{2}{*}{1} & 28 & f & WG & $\mathrm{N}$ & $>640$ & $\operatorname{Pr} 3$ & 33 & 190 & 0 & 14.4 & no \\
\hline & & & & Q & 40 & Pr3 & 0 & $<3$ & 2 & 6.9 & aza $150 \mathrm{mg}+$ pred $10 \mathrm{mg}$ \\
\hline \multirow[t]{2}{*}{2} & 63 & $\mathrm{~m}$ & WG & $\mathrm{N}$ & $>640$ & $\operatorname{Pr} 3$ & 39 & 149 & 2 & 11.5 & no \\
\hline & & & & $\mathrm{Q}$ & 80 & $\operatorname{Pr} 3$ & 0 & $<3$ & 2 & 6.5 & pred $12.5 \mathrm{mg}+\mathrm{CP} 75 \mathrm{mg}$ \\
\hline 3 & 85 & $\mathrm{~m}$ & WG & $\mathrm{N}$ & 160 & Pr3, HLE & 27 & 99 & 1 & 8.0 & no \\
\hline 4 & 36 & $\mathrm{f}$ & WG & $\mathrm{N}$ & 80 & $\operatorname{Pr} 3$ & 8 & 5 & 0 & 7.6 & no \\
\hline 5 & 38 & $\mathrm{f}$ & WG & $\mathrm{R}$ & $>640$ & $\operatorname{Pr} 3$ & 17 & 32 & 5 & 6.0 & $\mathrm{CP} 75 \mathrm{mg}$, pred $20 \mathrm{mg}$ \\
\hline 6 & 56 & $\mathrm{~m}$ & WG & $\mathrm{R}$ & 160 & Pr3 & 7 & 13 & 3 & 6.0 & no \\
\hline \multirow[t]{2}{*}{7} & 35 & $\mathrm{~m}$ & WG & $\mathrm{R}$ & 160 & $\operatorname{Pr} 3$ & 17 & 166 & 0 & 15.7 & pred $20 \mathrm{mg}$ \\
\hline & & & & Q & 20 & $\operatorname{Pr} 3$ & 0 & 11 & 1 & 7.5 & pred $2.5 \mathrm{mg}+$ aza $150 \mathrm{mg}$ \\
\hline \multirow[t]{2}{*}{8} & 63 & $\mathrm{~m}$ & WG & $\mathrm{R}$ & 320 & $\operatorname{Pr} 3$ & 11 & 47 & 2 & 4.4 & no \\
\hline & & & & Q & 20 & $\operatorname{Pr} 3$ & 0 & 44 & 3 & 8.7 & pred $10 \mathrm{mg}$ \\
\hline 9 & 76 & $\mathrm{f}$ & WG & $\mathrm{R}$ & 160 & MPO & 9 & 108 & 7 & 7.5 & no \\
\hline \multirow[t]{2}{*}{10} & 49 & $\mathrm{~m}$ & WG & $\mathrm{R}$ & 80 & Pr3 & 6 & 4 & 1 & 6.8 & no \\
\hline & & & & Q & 40 & $\operatorname{Pr} 3$ & 0 & 4 & 2 & 6.8 & no \\
\hline \multirow[t]{2}{*}{11} & 56 & $\mathrm{f}$ & WG & $\mathrm{N}$ & 320 & Pr3 & 19 & 55 & 0 & 18.6 & no \\
\hline & & & & Q & 20 & $\operatorname{Pr} 3$ & 0 & 3 & 1 & 8.0 & pred $10 \mathrm{mg}+\mathrm{CP} 100 \mathrm{mg}$ \\
\hline \multirow[t]{2}{*}{12} & 75 & $\mathrm{~m}$ & WG & $\mathrm{R}$ & 160 & Pr3, MPO & 7 & 17 & 2 & 5.8 & no \\
\hline & & & & Q & 40 & $\operatorname{Pr} 3, \mathrm{MPO}$ & 0 & 8 & 2 & 10.5 & pred $7.5 \mathrm{mg}$ \\
\hline 13 & 63 & f & WG & $\mathrm{R}$ & 80 & HLE & 3 & 8 & 2 & 5.2 & pred $5 \mathrm{mg}+$ aza $100 \mathrm{mg}$ \\
\hline \multirow[t]{2}{*}{14} & 48 & $\mathrm{f}$ & WG & $\mathrm{N}$ & 320 & Pr3 & 20 & 118 & 0 & 13.4 & no \\
\hline & & & & Q & 0 & $\operatorname{Pr} 3$ & 0 & $<3$ & 0 & 4.1 & aza $125 \mathrm{mg}+$ pred $20 \mathrm{mg}$ \\
\hline 15 & 54 & $\mathrm{f}$ & WG & $\mathrm{R}$ & 80 & $\operatorname{Pr} 3$ & 1 & 92 & 3 & 12.4 & no \\
\hline 16 & 51 & $\mathrm{f}$ & WG & $\mathrm{N}$ & $>640$ & MPO, HLE & 19 & 22 & 0 & 3.6 & no \\
\hline \multirow[t]{2}{*}{17} & 27 & $\mathrm{~m}$ & WG & $\mathrm{N}$ & 320 & $\operatorname{Pr} 3, \mathrm{LF}$ & 15 & 40 & 0 & 9.4 & no \\
\hline & & & & Q & 40 & $\mathrm{LF}$ & 0 & 52 & 1 & 9.7 & pred $20 \mathrm{mg}+$ aza $150 \mathrm{mg}$ \\
\hline \multirow[t]{2}{*}{18} & 44 & $\mathrm{~m}$ & WG & $\mathrm{N}$ & 320 & LF & 11 & 5 & 0 & 5.2 & no \\
\hline & & & & $\mathrm{Q}$ & 80 & LF & 0 & 5 & 2 & 8.1 & pred $32.5 \mathrm{mg}+\mathrm{CP} 50 \mathrm{mg}$ \\
\hline 19 & 79 & $\mathrm{f}$ & WG & $\mathrm{N}$ & 320 & $\operatorname{Pr} 3$ & 31 & 81 & 0 & 8.9 & no \\
\hline 20 & 23 & $\mathrm{f}$ & WG & $\mathrm{R}$ & 320 & MPO & 10 & $<3$ & 2 & 4.9 & aza $75 \mathrm{mg}$ \\
\hline 21 & 72 & f & WG & $\mathrm{R}$ & 160 & $\mathrm{MPO}$ & 12 & $<3$ & 2 & 7.8 & no \\
\hline 22 & 25 & $\mathrm{f}$ & WG & $\mathrm{N}$ & 40 & $\operatorname{Pr} 3$ & 20 & 105 & 0 & 13.1 & no \\
\hline 23 & 48 & $\mathrm{~m}$ & WG & $\mathrm{R}$ & 80 & Pr3 & 13 & 20 & 2 & 5.1 & no \\
\hline 24 & 69 & $\mathrm{~m}$ & WG & $\mathrm{N}$ & 320 & Pr3 & 28 & 23.0 & 0 & 178 & no \\
\hline
\end{tabular}

aza, azathioprine; CRP, C reactive protein (mg/l); CP, cyclophosphamide; HLE, human leucocyte elastase; MPO, myeloperoxydase; N, newly developed disease; nt, not tested; pred, prednisolone; Pr3, proteinase 3; Q, Quiescent disease; R, relapse; spec., ANCA specificity; titre, ANCA titre; WG, Wegener's granulomatosis; WBC, white blood cell count $\left(\times 10^{9} / 1\right)$.

tion between parameters was analysed by the Spearman rank correlation test. The paired Wilcoxon test was used to test differences between paired observations. These tests were performed by using GraphPad Instat2 Software. A two tailed $\mathrm{p}$ value $<0.05$ was considered to indicate statistical significance.

\section{Results}

PATIENTS

Twenty four patients (11 male, 13 female, mean age 53, range 23-85) with active

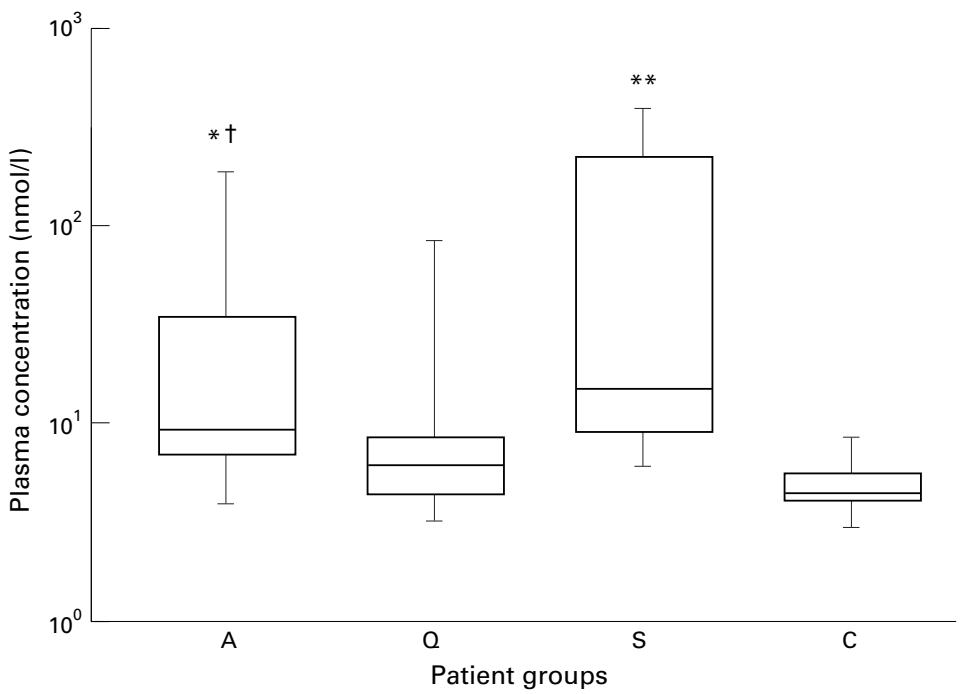

Figure 1 Box and whisker plots indicating the overall range (error bars), 25-75\% range (boxes), and median value (horizontal lines) of neopterin plasma concentrations in patients with WG (A: active disease, Q: quiescent disease) compared with concentrations in patients with sepsis $(S)$ and healthy controls $(C) .{ }^{\star} p<0.05,{ }^{\star}{ }^{\star} p<0.0001$ compared with healthy controls, $+p=0.074$ compared with quiescent disease.
WG-that is, either with newly developed disease $(n=12)$ or with relapsing disease $(n=$ 12) - were included in this study. Additionally, 10 of these patients were analysed also at the time of quiescent disease, when a complete remission had been obtained. Tables 3 and 4 give the patient characteristics.

SOLUBLE MARKERS OF MONOCYTE ACTIVATION Neopterin is secreted in large quantities by activated monocytes/macrophages upon activation. ${ }^{26}$ In patients with active disease neopterin values were higher compared with healthy controls $(\mathrm{p}<0.05)$ and tended to be higher compared with patients with quiescent disease $(p=0.07)$. Analysis of paired samples showed that plasma concentrations of neopterin in patients with active disease were not different compared with neopterin values during remission. In patients with sepsis plasma concentrations of neopterin were increased compared with healthy controls $(\mathrm{p}<0.0001)$ (fig 1).

IL6 concentrations did not differ between patients with active and quiescent disease, nor between patients with active disease and healthy controls, although a broad range of IL6

Table 4 Patient characteristics

\begin{tabular}{|c|c|c|}
\hline & $\begin{array}{l}\text { Active disease } \\
\text { median (range) }\end{array}$ & $\begin{array}{l}\text { Quiescent disease } \\
\text { median (range) }\end{array}$ \\
\hline Patients (n) & 24 & 10 \\
\hline Titre & $(40-640)$ & $(0-80)$ \\
\hline BVAS & $14 \quad(1-39)$ & $(0-0)$ \\
\hline $\mathrm{C}$ reactive protein & $36 \quad(2-190)$ & $5 \quad(2-52)$ \\
\hline WBC & $7.7(4.4-18.6)$ & $7.5(3.6-10.5)$ \\
\hline
\end{tabular}

BVAS, Birmingham Vasculitis Activity Score; WBC, white blood cell count. 

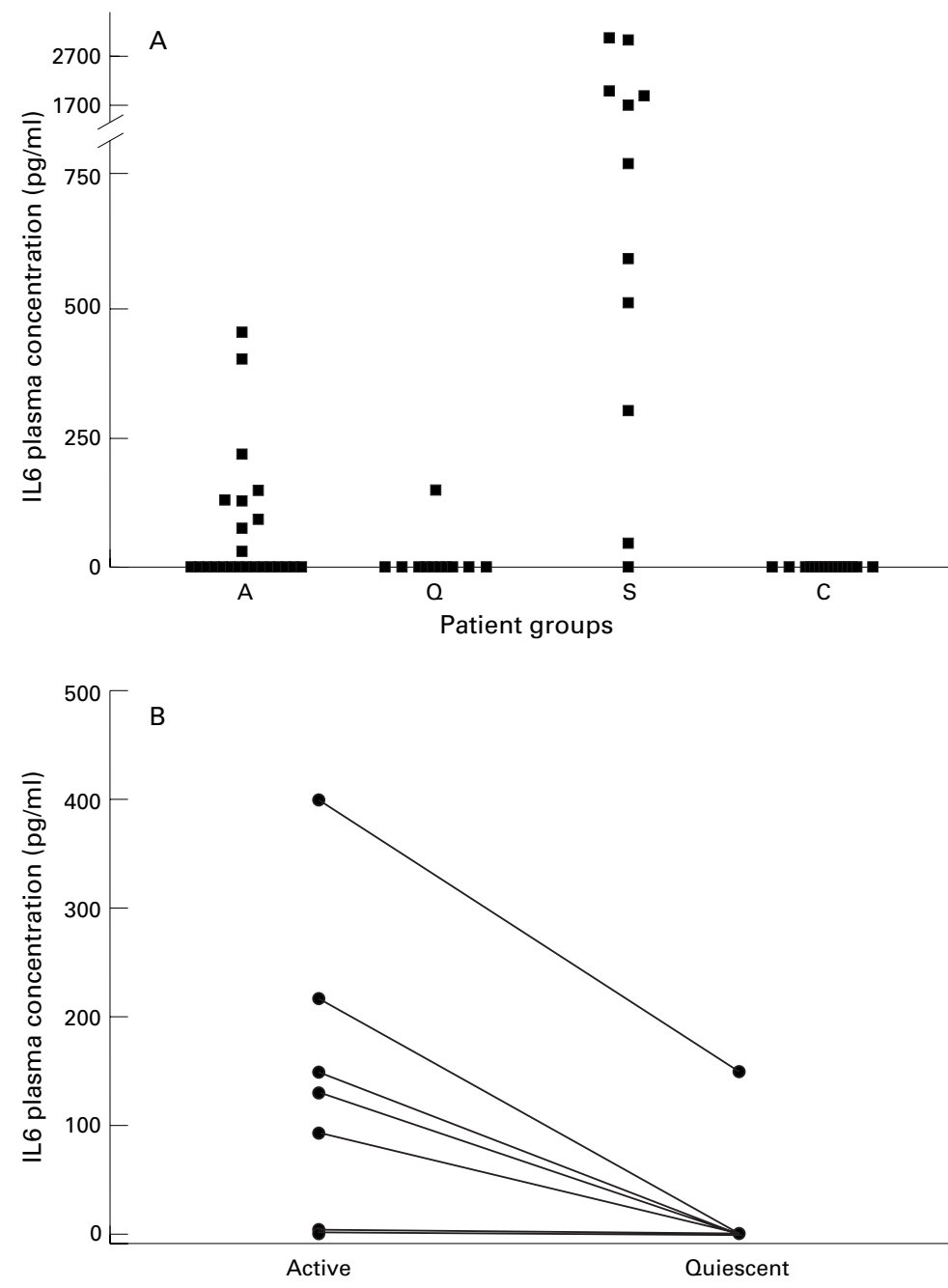

Figure 2 (A) A dot blot of the plasma concentrations of IL6 in 24 patients with WG (A: active disease, open squares represent 12 patients with newly diagnosed disease, closed squares represent 12 patients with relapsing disease, Q: quiescent disease, 10 patients) compared with concentrations in 12 patients with sepsis $(S)$ and healthy controls $(C)$. (B) Paired observations of IL 6 plasma concentrations in 10 patients with active and quiescent disease. $p<0.0001$. expression of CD63 on circulating monocytes from patients with WG.

Monocytes from patients with active disease showed a tendency to a higher expression of CD63 compared with healthy controls ( $\mathrm{p}=$ 0.08). Compared with patients in remission no differences were found. Analysis of paired data showed that CD63 expression in patients with active disease was not different compared with CD63 expression during remission. Monocytes from patients with sepsis showed a higher expression of CD63 than healthy controls ( $p<$ $0.0001)$. Results are shown in figure $3 \mathrm{~A}$.

Upon leucocyte activation the expression of CD11b, the $\alpha$ subunit of Mac-1, an adhesion molecule involved in cell adhesion and transmigration, is increased. Therefore, the expres-
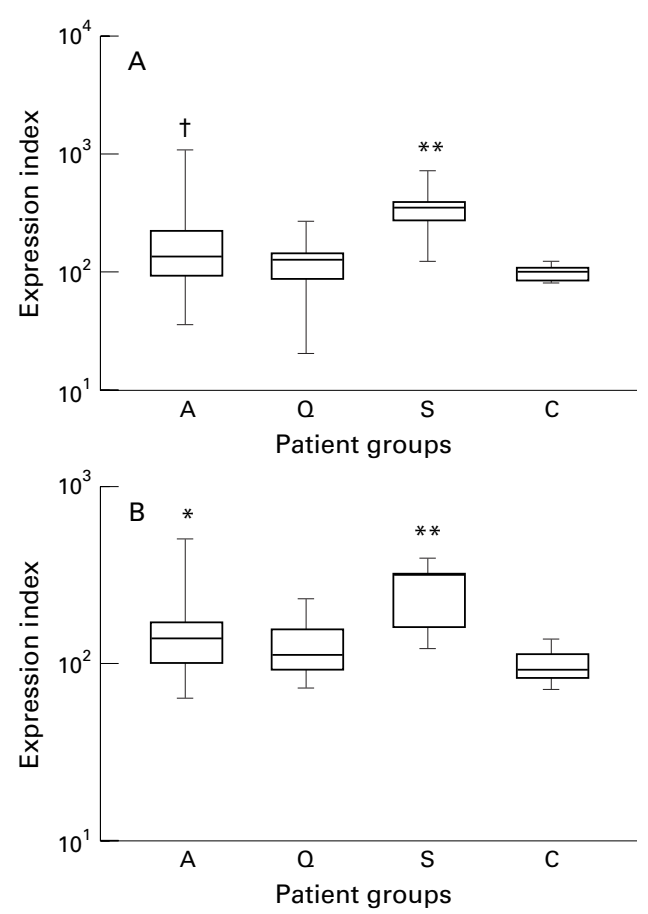

concentrations in patients with active disease was observed (fig 2A). Nine patients with active disease, one patient with quiescent disease and none of the controls had detectable concentrations of IL6 ( $p<0.0001$, active WG $v$ healthy controls). Patients who had detectable IL6 concentrations had higher CRP values $(\mathrm{p}<0.005)$ than patients without detectable IL6 plasma concentrations. In patients with newly diagnosed disease concentrations of IL6 tended to be higher compared with patients in remission $(p=0.06)$ and healthy controls $(p=0.06)$ (fig 2A). Analysis of paired samples showed that plasma concentrations of IL6 in patients with active disease were higher compared with IL6 levels during remission ( $\mathrm{p}<$ 0.0001 ) (fig 2B). In patients with sepsis plasma concentrations of IL6 were highly increased compared with healthy controls $(p<0.001)$.

EXPRESSION OF ACTIVATION MARKERS ON MONOCYTES

As the expression of CD63 is increased upon degranulation of monocytes, ${ }^{35}$ we studied the

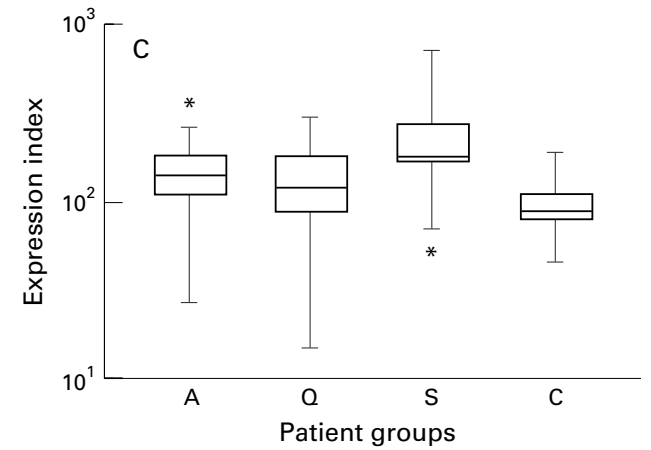

Figure 3 (A) Box and whisker plots of the surface expression CD63 on monocytes from patients with $W G(A$ : active disease, $Q$ : quiescent disease) compared with the expression on cells from patients with sepsis $(S)$ and healthy controls $(C) .+p=0.0787, \star \star p<0.0001$ compared with healthy controls. (B) Box and whisker plots of the surface expression of $C D 11$ on monocytes from patients with $W G$ (A: active disease, Q: quiescent disease) compared with the expression on cells from patients with sepsis (S) and healthy controls $(C) .{ }^{\star} p<0.05,{ }^{\star} p<0.0001$ compared with healthy controls. (C) Box and whisker plots of the surface expression of CD64 on monocytes from patients with $W G$ (A: active disease, Q: quiescent disease) compared with the expression on cells from patients with sepsis (S) and healthy controls $(C) .{ }^{\star} p<0.05$ compared with healthy controls. 
sion of CD11b on monocytes was measured to analyse monocyte activation.

CD1 1b expression was increased on monocytes from patients with active disease compared with monocytes from healthy controls ( $p$ $<0.05$ ), but did not differ from the expression on monocytes from patients with quiescent disease. Analysis of paired data showed that CD11b expression in patients with active disease was not different compared with CD11b expression during remission. CD $11 b$ expression on monocytes from patients with sepsis was clearly increased compared with healthy controls $(\mathrm{p}<0.0001)$. Data are represented in figure 3B.

VLA-4 expression on monocytes did not differ between patients with various stages of disease activity and healthy controls. In addition, the expression of VLA-4 on monocytes from patients with sepsis, was not significantly different compared with healthy controls.

The first Fc gamma receptor, CD64, is expressed constitutively on monocytes and its expression is increased upon activation. ${ }^{36}$ Compared with healthy controls, patients with active disease had an increased expression of CD64 on monocytes $(\mathrm{p}<0.05)$. When compared with patients in remission, no differences in CD64 expression were found. Analysis of paired data showed that CD64 expression in patients with active disease was not different compared with CD64 expression during remission. Monocytes from patients with sepsis showed increased expression of CD64 ( $p<$ 0.05) compared with healthy controls. Results are shown in figure $3 \mathrm{C}$.

EXPRESSION OF ANCA ANTIGENS ON MONOCYTES On monocytes the expression of ANCA antigens is increased in vitro when cells are primed with cytokines. ${ }^{13}$ To evaluate whether circulating monocytes in vivo also express ANCA antigens on their cell surface, we measured the expression of ANCA antigens on monocytes of patients and controls.

Circulating monocytes from patients and healthy controls expressed MPO and Pr3, whereas the expression of HLE was low or absent. The expression of Pr3, MPO, or HLE on monocytes did not differ significantly between the various groups of patients and healthy controls, although individual patients had increased expression of $\operatorname{Pr} 3$ and/or MPO. A flow cytometry histogram of $\operatorname{Pr} 3$ and MPO expression on monocytes from a patient with active disease is shown in figure 4 . Monocytes
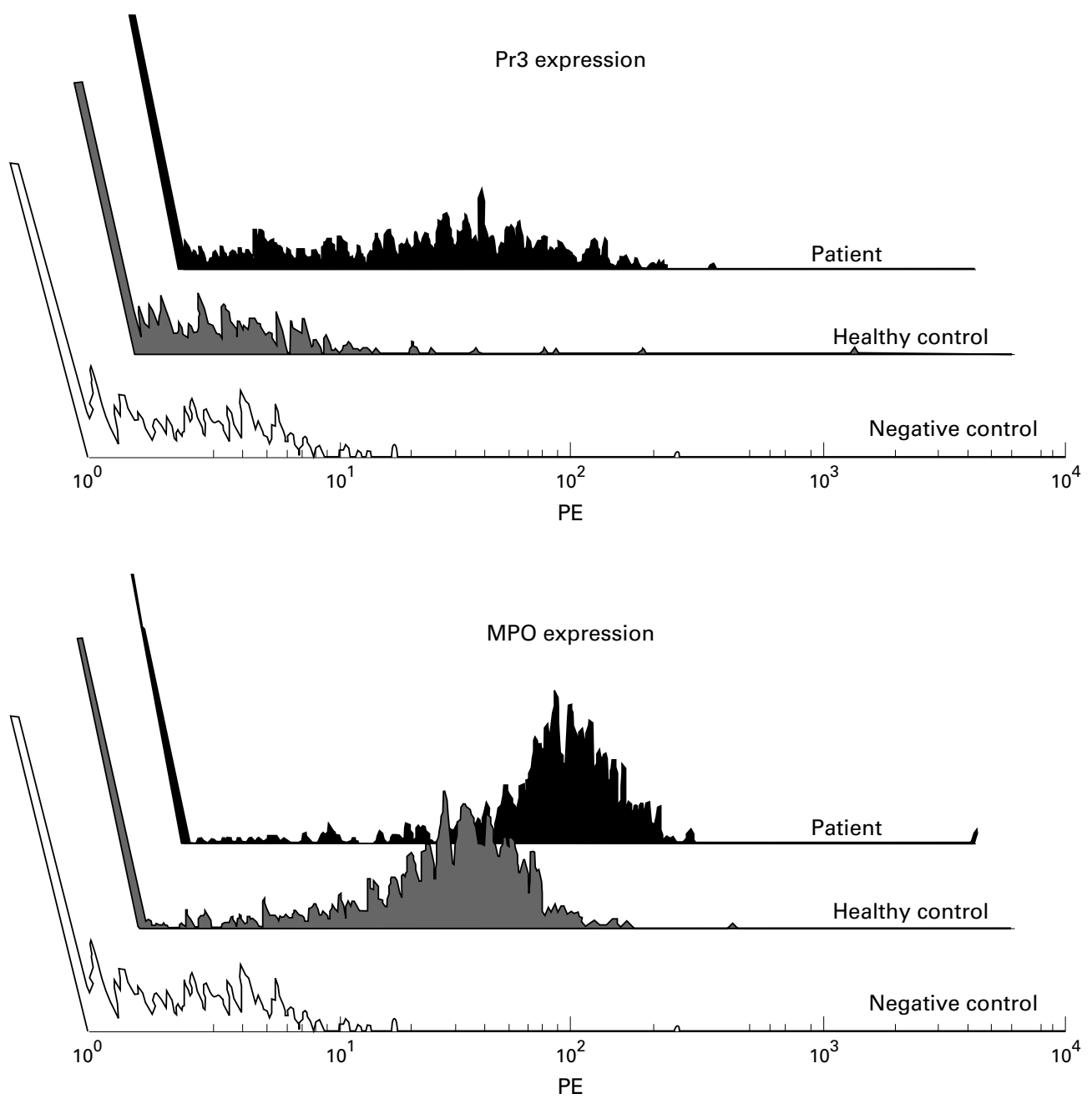

Figure 4 Flow cytometry histogram representing membrane expression of $\operatorname{Pr} 3$ and MPO and the negative control staining on monocytes from a patient with active WG and a healthy control. Cell count and the mean fluorescence intensity (MFI) are depicted on the $y$ and $x$ axes respectively. 
from patients with sepsis showed an increased expression of $\operatorname{Pr} 3$ compared with the expression on monocytes from healthy controls $(\mathrm{p}<0.05)$.

\section{CORRELATIONS WITH MARKERS OF DISEASE} ACTIVITY

Correlations were found between soluble markers of monocyte activation and disease activity: Both neopterin levels and IL6 levels of patients with detectable IL6 levels correlated with the BVAS score $(r=0.3664, \mathrm{p}<0.05$ and $r=0.8096, \mathrm{p}<0.0001$, respectively) and CRP values $(r=0.4437, \mathrm{p}<0.001$, and $r=0.7385$, $\mathrm{p}<0.001)$.

In addition, membrane expression of CD63 on circulating monocytes correlated with the BVAS score $(r=0.3937, \mathrm{p}<0.05)$ and CRP values $(r=0.4489, \mathrm{p}<0.01)$ (fig 5$)$.

Next, we compared soluble markers of monocyte activation with cellular expression of activation markers on circulating monocytes. Surface expression of CD63 correlated with IL6 $(r=0.4393, \mathrm{p}<0.05)$ but not with levels of neopterin.

To detect a possible effect of treatment, we analysed whether there was a relation between
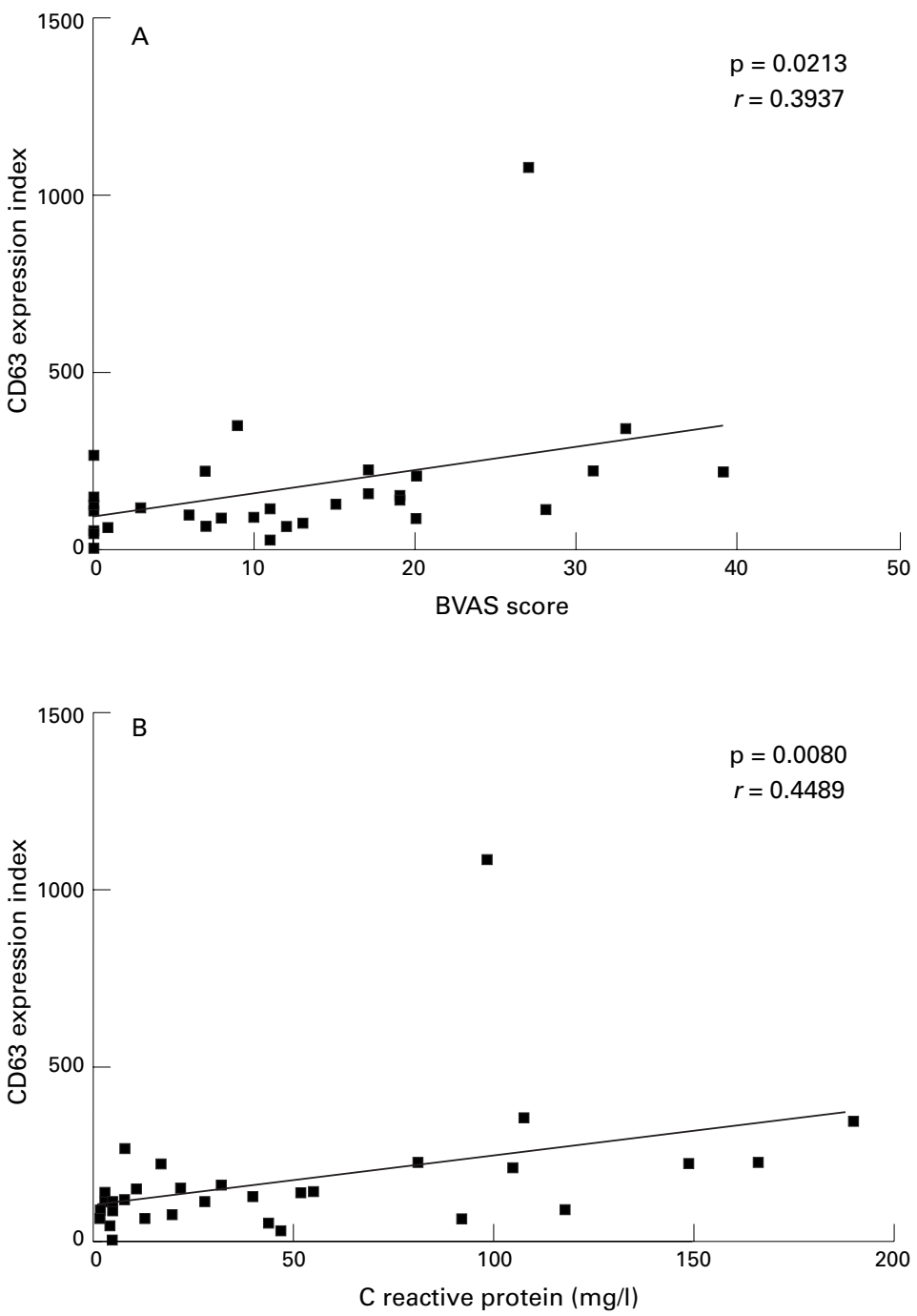

Figure 5 Surface expression of CD63 on monocytes (expressed as expression index, see methods) correlate with disease activity as expressed by the BVAS score (A) or CRP values (B). the use of corticosteroids or cytotoxic agents, or both, on the one hand and the levels of expression of activation markers on the other hand. No such a relation could be detected.

\section{Discussion}

In this study we demonstrated monocyte activation in patients with WG by analysing soluble products of activated monocytes, in particular neopterin and IL6, and the membrane expression of activation markers on circulating monocytes. Plasma concentrations of neopterin as well as surface expression of CD11b and CD64 on circulating monocytes were increased in patients with active disease compared with healthy controls. In addition, levels of neopterin and IL6 and the surface expression of CD63 on monocytes correlated with disease activity. Thus, monocytes are already activated in the circulation, and may eventually participate in granuloma formation and generalised vasculitis in patients with WG.

In this study, we found increased levels of neopterin in patients with active WG. As neopterin plasma concentrations may be influenced by renal clearance, ${ }^{37}$ we also calculated neopterin/serum creatinine ratios and still found increased levels of neopterin in patients with WG compared with healthy controls that correlated with parameters of disease activity (data not shown). Increased plasma concentrations of neopterin in patients with WG correlating with disease activity were also demonstrated by Nassonov et al. ${ }^{38}$ In addition, Nassonov et al showed that during follow up neopterin concentrations paralleled the course of disease activity in these patients.

Plasma concentrations of IL6 did not differ between the various stages of disease. This was mainly because of the broad range of IL6 concentrations in patients with active disease. Nine patients with active disease had detectable levels of IL6 and these concentrations were correlated with disease activity. In addition, patients who had detectable IL6 levels had higher CRP values than patients without detectable IL6 plasma levels. Furthermore, in patients with newly diagnosed disease levels of IL6 tended to be higher compared with patients in remission and healthy controls. During follow up plasma levels in these patients decreased to normal values.

Thus, monocyte activation, as reflected by the release of soluble products, corresponds to the clinical expression of the disease.

Plasma levels of neopterin and IL6 reflect the state of activation of both extravasated monocytes/macrophages as well as circulating monocytes, whereas the surface expression of activation markers reflect the state of activation of circulating monocytes only.

Therefore, the extent of cell activation of circulating monocytes was also analysed in this study by the measurement of surface expression of several monocyte activation markers. The expression of CD63 tended to be increased on monocytes from patients with active disease compared with healthy controls, indicating that degranulation of azurophilic granules, in which MPO and Pr3 are stored, 
has occurred. Degranulation of azurophilic granules is thought to be the initial step for the expression of ANCA antigens on the cell surface of both neutrophils and monocytes, but also for "planting" those antigens at the endothelial cell surface. In addition, if degranulation occurs in the vicinity of the endothelial lining, this may result in endothelial damage because of the release of lytic enzymes and oxygen radicals. In this study, we demonstrated that the extent of degranulation, as measured by CD63 expression, correlated with disease activity.

Upon activation, the expression of several adhesion molecules, including CD11b and VLA-4, on monocytes increases. CD11b is expressed on most leucocytes, whereas VLA-4 is mainly expressed on monocytes, lymphocytes and eosinophils. ${ }^{39}$ VLA-4 is a ligand for the vascular cell adhesion molecule, VCAM-1, on endothelial cells. The interaction of VLA-4 with VCAM-1 is thought to be of importance for adhesion to, and transmigration through the endothelium, in particular by monocytes. Transmigrated monocytes may participate in granuloma formation. We found an increased expression of CD $11 \mathrm{~b}$ on circulating monocytes from patients with active disease, but the expression of VLA-4 on monocytes was not increased. During activation, integrins, like CD1 $1 \mathrm{~b}$ and VLA-4, demonstrate increased affinity towards their respective ligands. ${ }^{40} \mathrm{CD} 11 \mathrm{~b}$, however, also demonstrates an increased avidity, as the number of CD11b molecules on the cell surface increases upon cell activation. In our study, the anti-CD $11 \mathrm{~b}$ and the anti- VLA-4 monoclonal antibody used for membrane detection could not distinguish between "high" and "low" affine integrins. As fully activated monocytes, expressing high affine VLA-4 and/or CD $11 \mathrm{~b}$, adhere to the endothelium, these adherent cells cannot be detected anymore in the circulation. Furthermore, Stegeman et $a l^{41}$ demonstrated increased levels of soluble VCAM in patients with WG, whereas levels of soluble ICAM-1 were only slightly increased. This may indicate that, in patients with WG, the VLA-4/ VCAM-1 interaction plays a more important part than the CD11b/ICAM-1 interaction.

The expression of CD64 (the first Fc $\gamma$ receptor) was increased on monocytes from patients with active disease compared with healthy controls. Fc gamma receptors are essential for monocyte activation by ANCA as was recently demonstrated by Ralston et $a l^{13}$ when using $\mathrm{Fab}$ or $\mathrm{F}(\mathrm{ab}) 2$ fragments of anti-Pr3 antibodies. As monocytes themselves contain the ANCA antigens Pr3 and MPO, these antigens can be expressed at the cellsurface. In vitro, this has been recently demonstrated by Ralton et al. ${ }^{13}$

In this study, we found that the expression of CD63, CD11b and CD64 on circulating monocytes from patients with active WG were increased compared with healthy controls, but were lower than the expression on monocytes from patients with sepsis. Thus, compared with patients with sepsis, monocyte activation in patients with vasculitis is mild.
The stronger correlations found between soluble markers of monocyte activation and disease activity in patients with WG may indicate that the activation of tissue macrophages rather than circulating, peripheral blood monocytes plays an important part in the pathogenesis of this disease.

This study demonstrated that, in vivo, monocytes are capable of expressing $\operatorname{Pr} 3$ and MPO. Pr3 and MPO expression on monocytes is essential for interaction with ANCA and subsequent activation by ANCA. Indeed, primed monocytes produce increased levels of MCP-1 and IL8 upon stimulation with ANCA in vitro. ${ }^{13}{ }^{14}$ Both MCP-1 and IL8 are potent chemoattractants for monocytes and neutrophils. They may, therefore, play a part in the recruitment of (activated circulating) monocytes and neutrophils into evolving granulomas. ${ }^{12}$ Furthermore, IL8 may stimulate PMNs to express $\operatorname{Pr} 3,{ }^{8}$ thereby amplifying the effector phase in vasculitis. Expression of ANCA antigens on circulating monocytes can, however, be observed in the presence of ANCA in patients without active disease - that is, during quiescent disease. Therefore, other additional factors are probably involved in the activation of monocytes by ANCA.

From our data we hypothesise that monocytes in patients with WG may be primed as a result of stimulation by proinflammatory cytokines, which are locally released upon infections, which are often observed in these patients. ${ }^{42-44}$ Alternatively, priming of circulating monocytes may also result from cytokines released from inflammatory sites. Subsequently, primed monocytes are systemically activated because of stimulation by ANCA. These activated circulating monocytes adhere to and transmigrate through endothelial cells, which are also activated in WG. ${ }^{45}$ These transmigrated activated monocytes, then participate in granuloma formation and amplify inflammation by the production of MCP-1 or IL8, or both. These chemoattractants then attract a new influx of leucocytes.

In conclusion, disease activity in WG correlates with the extent of activation of monocytes, compatible with their role in the pathophysiology of this disease.

The authors wish to thank M G Huitema, W W Oost-Kort, I Bouwman, and G Mesander for their technical assistance, Drs J Zijlstra, C A Stegeman, and C F A Franssen for their clinical input, and Dr P C Limburg for his valuable advice. Funding: this study was performed with the aid of departmental funds.

1 Kallenberg CGM, Brouwer E, Weening JJ, Cohen Tervaert JW. Anti-neutrophil cytoplasmic antibodies: current diagnostic and pathophysiological potential. Kidney Int $1994 ; 46: 1-15$

2 Cohen Tervaert JW, Huitema MG, Hene RJ, Sluiter WJ, The TH, van der Hem GK, et al. Prevention of relapses in Wegener's granulomatosis by treatment based on antineutrophil cytoplasmic antibody titre. Lancet 1990;336:70911 .

3 Falk RJ, Terrell RS, Charles LA, Jennette JC. Antineutrophil cytoplasmic autoantibodies induce neutrophils to degranulate and produce oxygen radicals in vitro. Proc Natl Acad Sci USA 1990;87:4115-19.

4 Charles LA, Caldas ML, Falk RJ, Terrell RS, Jennette JC. Antibodies against granule proteins activate neutrophils in vitro. J Leukoc Biol 1991;50:539-46.

5 Savage CO, Pottinger BE, Gaskin G, Pusey CD, Pearson JD. Autoantibodies developing to myeloperoxidase and proteinase 3 in systemic vasculitis stimulate neutrophil cytotoxicity toward cultured endothelial cells. Am J Pathol 1992;141:335-42. 
6 Mayet WJ, Meyer zum Buschenfelde KH. Antibodies to proteinase 3 increase adhesion of neutrophils to human

7 Ewert BH, Becker ME, Jennette JC, Falk RJ. Antimyeloperoxidase antibodies induce neutrophil adherence to cultured human endothelial cells. Ren Fail 1995;17:125-33.

8 Csernok E, Ernst M, Schmitt W, Bainton DF, Gross WL. Activated neutrophils express proteinase 3 on their plasma membrane in vitro and in vivo. Clin Exp Immuno 1994;95:244-50.

9 Porges AJ, Redecha PB, Kimberly WT, Csernok E, Gross WL, Kimberly RP. Anti-neutrophil cytoplasmic antibodies
engage and activate human neutrophils via Fc gamma RIIa. J Immunol 1994;153:1271-80.

10 Mulder AH, Heeringa P, Brouwer E, Limburg PC, Kallenberg CGM. Activation of granulocytes by anti-neutrophil cytoplasmic antibodies (ANCA): a FC gamma RIIdependent process. Clin Exp Immunol 1994;98:270-8.

11 Rastaldi MP, Ferrario F, Tunesi S, Yang L, D'Amico G. Intraglomerular and interstitial leukocyte infiltration, adhesion molecules, and interleukin-1 a expression in 15 cases of
antineutrophil cytoplasmic autoantibody-associated renal antineutrophil cytoplasmic autoantibody-assoc
vasculitis. Am J Kidney Dis 1996;27:48-57.

12 Pike PC. The role of the monocyte and macrophage in the pathogenisis of vasculitis. In: LeRoy EC, ed. Systemic vasculitis. The biological basis. New York: Marcel Dekker, 1992:129-47.

13 Ralston DR, Marsh CB, Lowe MP, Wewers MD. Antineutrophil cytoplasmic antibodies induce monocyte IL-8 release. Role of surface proteinase 3, alpha1-antitrypsin, and Fcgamma receptors. J Clin Invest 1997;100:1416-24

14 Casselman BL, Kilgore KS, Miller BF, Warren JS. Antibodies to neutrophil cytoplasmic antigens induce monocyte chemoattractant protein-1 secretion from human monocytes. J Lab Clin Med 1995;126:495-502.

15 Riecken B, Gutfleisch J, Schlesier M, Peter HH. Impaired granulocyte oxidative burst and decreased expression of leucocyte adhesion molecule-1 (LAM-1) in patients with Wegener's granulomatosis. Clin Exp Immunol 1994;96: 43-7.

16 Haller H, Eichhorn J, Pieper K, Goebel U, Luft FC. Circulating leukocyte integrin expression in Wegener's granulomatosis. J Am Soc Nephrol 1996;7:40-8.

17 Ziegler EJ, Fisher CJ Jr, Sprung CL, Straube RC, Sadoff JC, Foulke GE, et al. Treatment of gram-negative bacteremia and septic shock with HA-1A human monoclonal antibody against endotoxin. A randomized, double-blind, placebocontrolled trial. The HA-1A Sepsis Study Group. N Engl J Med 1991;324:429-36.

18 Fauci AS, Haynes BF, Katz P, Wolff SM. Wegener's granulomatosis: prospective clinical and therapeutic expe-
rience with 85 patients for 21 years. Ann Intern Med 1983; 98:76-85.

19 Leavitt RY, Fauci AS, Bloch DA, Michel BA, Hunder GG, Arend WP, et al. The American College of Rheumatology 1990 criteria for the classification of Wegener's granulomatosis. Arthritis Rheum 1990;33:1101-7.

20 Stegeman CA, Cohen Tervaert JW, de Jong PE, Kallenberg CGM. Trimethoprim-sulfamethoxazole (co-trimoxazole) CGM. Trimethoprim-sulfamethoxazole (co-trimoxazole) for the prevention of relapses of Wegener's granulomatosis. Dutch Co-Trimoxazole

21 Luqmani RA, Bacon PA, Moots RJ, Janssen BA, Pall A, Emery $\mathrm{P}$, et al. Birmingham Vasculitis Activity Score (BVAS) in systemic necrotizing vasculitis. Q J Med 1994;87:671-8

22 Exley AR, Bacon PA, Luqmani RA, Kitas GD, Gordon C, Savage CO, et al. Development and initial validation of the Vasculitis Damage Index for the standardized clinical assessment of damage in the systemic vasculitides. Arthritis Rheum 1997;40:371-80.

23 Cohen Tervaert JW, Mulder L, Stegeman C, Elema J, Huitema $\mathrm{M}$, The $\mathrm{H}$, et al. Occurrence of autoantibodies to human leucocyte elastase in Wegener's granulomatosis and other inflammatory disorders. Ann Rheum Dis 1993;52: other in $115-20$.

24 Cohen Tervaert JW, Goldschmeding R, Elema JD, van der Giessen M, Huitema MG, van der Hem GK, et al. Autoan-
tibodies against myeloid lysosomal enzymes in crescentic tibodies against myeloid lysosomal enzymes in cr

25 Mulder AH, Broekroelofs J, Horst G, Limburg PC, Nelis GF, Kallenberg CGM. Anti-neutrophil cytoplasmic antibodies (ANCA) in inflammatory bowel disease: characterization and clinical correlates. Clin Exp Immunol 1994;95: 490-7.

26 Fuchs D, Weiss G, Reibnegger G, Wachter H. The role of neopterin as a monitor of cellular immune activation in transplantation, inflammatory, infectious, and malignant diseases. Crit Rev Clin Lab Sciences 1992;29:307-41.

27 Samsonov MY, Tilz GP, Egorova O, Reibnegger G, Nassonov EL, Nassonova VA, et al. Serum soluble markers of immune activation and disease activity in systemic lupus erythematosus. Lupus 1995;4:29-32.

28 Haupt W, Hohenberger W, Klein P, Christou NV. Detection of neopterin, interleukin 6 and acute phase proteins as parameters of potential monocyte activation in preoperative patients. Infection 1995;23:263-6.

29 Horneff G, Sack U, Kalden JR, Emmerich F, Burmester GR. Reduction of monocyte-macrophage activation markers upon anti-CD4 treatment. Decreased levels of IL-1, IL-6, neopterin and soluble CD14 in patients with rheumatoid arthritis. Clin Exp Immunol 1993;91:207-13.

30 Helle M, Boeye L, Groot Ed, Vis Ad, Aarden L. Sensitive Elisa for interleukin-6: Detection of IL-6 in biological 138:47-56.

31 Kuijpers TW, Tool AT, van der Schoot CE, Ginsel LA, Onderwater JJ, Roos D, et al. Membrane surface antigen expression on neutrophils: a reappraisal of the use of expression on neutrophils: a reappraisal of the use of 1105-11.

32 Muller Kobold AC, Kallenberg CGM, Cohen Tervaert JW. Leukocyte membrane expression of proteinase 3 correlates with disease activity in patients with Wegener's granulomatosis. Br J Rheumatol 1998;37:901-7.

33 Liote F, Boval-Boizard B, Weill D, Kuntz D, Wautier JL. Blood monocyte activation in rheumatoid arthritis: increased monocyte adhesiveness, integrin expression, and cytokine release. Clin Exp Immunol 1996;106:13-9.

34 Sladek TL, Jacobberger JW. Flow cytometric titration of retroviral expression vectors: comparison of methods for analysis of immunofluorescence histograms derived from cells expressing low antigen levels. Cytometry 1993;14:2331 .

35 Metzelaar MJ, Wijngaard PL, Peters PJ, Sixma JJ, Nieuwenhuis HK, Clevers HC. CD63 antigen. A novel lysosomal membrane glycoprotein, cloned by a screening procedure for intracellular antigens in eukaryotic cells. J Biol Chem 1991;266:3239-45.

36 Valerius T, Repp R, de Wit TP, Berthold S, Platzer E, Kalden JR, et al. Involvement of the high-affinity receptor for IgG (FC gamma RI; CD64) in enhanced tumor cell cytotoxicity of neutrophils during granulocyte colonycytotoxicity of neutrophils during granulocyte
stimulating factor therapy. Blood 1993;82:931-9.

37 Aulitzky WE, Tilg H, Niederwieser D, Riccabona G, Obendorf L, Margreiter R, et al. Comparison of serum neopterin levels and urinary excretion in renal allograft recipients. Clin Nephrology 1988;29:248-52.

38 Nassonov E, Samsonov M, Beketova T, Semenkova L, Wachter H, Fuchs D. Serum neopterin concentrations in Wegener's granulomatosis correlate with vasculitis activity. Clin Exp Rheumatol 1995; 13:353-6.

39 Wayner EA, Garcia Pardo A, Humphries MJ, McDonald JA, Carter WG. Identification and characterization of the $\mathrm{T}$ lymphocyte adhesion receptor for an alternative cell attachment domain (CS-1) in plasma fibronectin. J Cell Biol 1989;109:1321-30.

40 Springer TA. Adhesion receptors of the immune system. Nature 1990;346:425-34.

41 Stegeman CA, Cohen Tervaert JW, Huitema MG, de Jong PE, Kallenberg CGM. Serum levels of soluble adhesion molecules intercellular adhesion molecule 1, vascular adhesion molecule 1 , and E-selectin in patients with Wegener's granulomatosis. Arthritis Rheum 1994;37:1228-35.

42 Pinching AJ, Rees AJ, Pussell BA, Lockwood CM, Mitchison RS, Peters DK. Relapses in Wegener's granulomatosis: the role of infection. BMJ 1980;281: 836-8.

43 Falk RJ, Hogan S, Carey TS, Jennette JC. Clinical course of anti-neutrophil cytoplasmic autoantibody-associated glomerulonephritis and systemic vasculitis. The Glomerular Disease Collaborative Network. Ann Intern Med 1990; 113:656-63.

44 Stegeman CA, Cohen Tervaert JW, Sluiter WJ, Manson WL, de Jong PE, Kallenberg CGM. Association of chronic nasal carriage of Staphylococcus aureus and higher relapse rates in Wegener granulomatosis. Ann Intern Med 1994;120:12-17.

45 Cohen Tervaert JW, Kallenberg CGM. Cell adhesion molecules in vasculitis. Curr Opin Rheumatol 1997;9:1625 . 